\title{
Flow and Noise Characteristics of Centrifugal Fan under Different Stall Conditions
}

\author{
Lei Zhang, Shoufang Liang, and Chenxing $\mathrm{Hu}$ \\ Key Laboratory of Condition Monitoring and Control for Power Plant Equipment, North China Electric Power University, \\ Ministry of Education, Baoding 071003, China
}

Correspondence should be addressed to Lei Zhang; ncepu_zhanglei@163.com

Received 29 November 2013; Accepted 9 April 2014; Published 22 May 2014

Academic Editor: Shaoyong Lai

Copyright (C) 2014 Lei Zhang et al. This is an open access article distributed under the Creative Commons Attribution License, which permits unrestricted use, distribution, and reproduction in any medium, provided the original work is properly cited.

\begin{abstract}
An implicit, time-accurate 3D Reynolds-averaged Navier-Stokes (RANS) solver is used to simulate the rotating stall phenomenon in a centrifugal fan. The goal of the present work is to shed light on the flow field and particularly the aerodynamic noise at different stall conditions. Aerodynamic characteristics, frequency domain characteristics, and the contours of sound power level under two different stall conditions are discussed in this paper. The results show that, with the decrease of valve opening, the amplitude of full pressure and flow fluctuations tends to be larger and the stall frequency remains the same. The flow field analysis indicates that the area occupied by stall cells expands with the decrease of flow rate. The noise calculation based on the simulation underlines the role of vortex noise after the occurrence of rotating stall, showing that the high noise area rotates along with the stall cell in the circumferential direction.
\end{abstract}

\section{Introduction}

As the power source of the air and gas system in the thermal power plant, the operation status of the centrifugal fan is directly related to the safe and economic operation of the power plant. Rotating stall in the centrifugal fan is a local instabilities phenomenon in which one or more cells propagate along the blade row in the circumferential direction. The nonuniform flow, the so-called stall cell, rotates as a fraction of the shaft speed, typically between $20 \%$ and $70 \%$. This running mode is responsible for strong vibrations which could damage the blades [1]. Meanwhile, it will increase the aerodynamic noise.

In order to reveal the generation mechanism of rotating stall, lots of models and theories have been proposed since the 1960s. Especially, experimental methods were widely used to illustrate the characteristics of internal flow field during stall. Lennemann and Howard discussed the causes of stall cells in low flow rate condition through the hydrogen bubble flow visualization method [2]. Lucius and Brenner experimentally studied the speed variation of a centrifugal pump in rotating stall stage [3]. For the centrifugal turbomachine, multiple factors can affect the characteristics of stall. Vaneless diffuser, for example, has significant influence on stall. Hasmatuchi et al. experimentally investigated the effect of blowing technology on the flow field of a centrifugal pump under rotating stall [4]. Rodgers conducted an experimental research on rotating stall in a centrifugal compressor with a vaneless diffuser and found that the stall margin can be improved through adjusting the expansion pressure factor [5]. Abidogun carried out an experiment to investigate the influence of vaneless diffuser on the stall characteristics. The results showed that increasing the length of diffuser can improve the rotating speed of stall, and the change of width showed no effect on stall [6].

Further efforts were made to study the stall inception in order to avoid the occurrence or minimize the effect of stall. As well accepted, two types of stall inception proposed by Camp and Day modal wave inception and spike inception were investigated experimentally [7]. Leinhos et al. studied development process of stall inception under instantaneous inflow distortion in an axial compressor [8].

With the rapid development of computer technology, numerical simulation has become an important method for flow field research of turbomachine under rotating stall conditions. Gourdain et al. investigated the ability of an unsteady flow solver to simulate the rotating stall phenomenon in an 
axial compressor and found that it was necessary to take the whole geometry into consideration to correctly predict the stall frequency [1]. Choi et al. investigated the effects of fan speed on rotating stall inception; the results showed that, at $60 \%$ speed (subsonic), tip leakage flow spillage occurred successively in the trailing blades of the mis-staggered blades [9]. Zhang et al. numerically studied the stall inception in a centrifugal fan, and the results showed that the stall inception experienced probably 50 rotor cycles developing into a stall group. The inception showed significant modal waveform. The importance of volute for generation of stall inception was illustrated through flow field analysis [10].

Aerodynamic noise is mainly caused by vortex and flow separation. So the unsteady behavior of rotating stall may have an influence on the noise of centrifugal fan. In capturing the physical mechanism of the fan noise associated with rotating stall, the primary work is to characterize the noise. During the 1960s, the interaction between noise and turbulence was discussed by Powell, and the vortex sound theory was proposed to explain the generation of acoustic sound. Then, Lighthill made a breakthrough in aerodynamic noise theory research by proposing the acoustic analogy [11]. Based on these works, Díaz et al. put forward a prediction of the tonal noise generation in an axial flow fan, and the noise level in the blower far-field region was estimated by means of acoustic analogy [12]. Scheit et al. analyzed the far-field noise in a centrifugal fan with an acoustic analogy method and presented design guidelines to optimize the radiated noise of the impeller [13]. The global control of subsonic axial fan at the blade passing frequency was also discussed by Gérard et al. [14]. He aimed at cancelling the tonal noise by using a single loudspeaker in front of the fan with a single-inputsingle-output adaptive feedforward controller. According to Ouyang et al's work, the far-field noise generated by crossflow fan with different impellers was measured and it showed the great influence of blade angles on the inflow pattern [15]. Based on the previous research, a new method to predict the fan noise and performance is developed by Lee et al., and through an acoustic analogy, the acoustic pressures from the unsteady force fluctuations of the blades are obtained [16].

In summary, a wide range of flow characteristics on rotating stall in compressor have been investigated and the researches concentrated on stall inception. The present work focuses on two aspects: simulation of the rotating stall phenomenon with a 3D flow solver and seeking the deep physical mechanism of this instability in a centrifugal fan. The numerical method is presented with the model and the particular boundary conditions are used. Results from the whole geometry simulation are then analyzed. In the first part, aerodynamic characteristics and frequency domain characteristics of the centrifugal fan under different stall conditions are analyzed. In the second part, the velocity vector field distributions in the centrifugal fan are discussed. Finally, noise characteristics of the centrifugal fan under different stall conditions are studied. And the noise characteristics during the circumferential propagation of stall cells are also discussed.

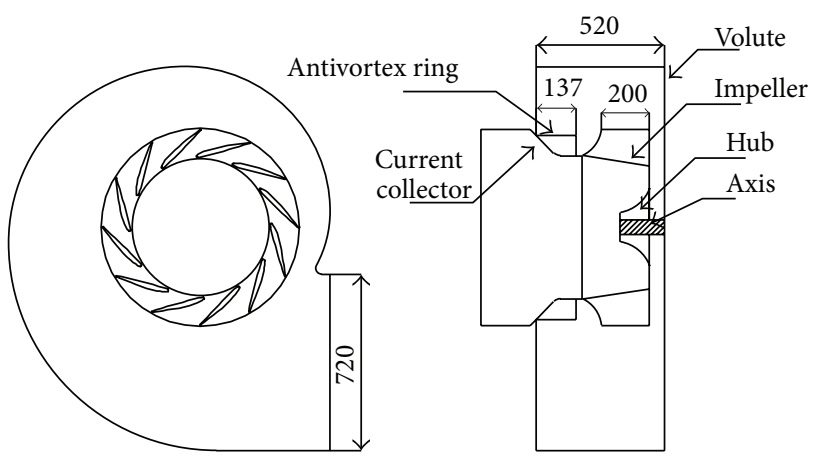

FIgURE 1: Fan structure diagram.

\section{Centrifugal Fan Description}

The configuration of centrifugal fan studied in this work is shown in Figure 1. It is composed of current collector, impeller with 12 airfoil blades, and the volute. The inlet and outlet diameter of the impeller are $568 \mathrm{~mm}$ and $800 \mathrm{~mm}$, respectively. The inlet and outlet width of impeller are $271 \mathrm{~mm}$ and $200 \mathrm{~mm}$, respectively. The nominal rotation speed is $1450 \mathrm{rpm}$. The volute tongue gap is $1 \%$ of the impeller outlet diameter. The width of the rectangular volute is $520 \mathrm{~mm}$, and a simple antivortex ring is set inside the volute to reduce the generation of vortex. At the design operating point, the volume flow is $6.32 \mathrm{~m}^{3} / \mathrm{s}$ and the full pressure is $1870 \mathrm{~Pa}$.

\section{Numerical Simulation}

3.1. Flow Solver and Boundary Conditions. Since the simulation of rotating stall requires a time-accurate method, the flow is simulated by solving the unsteady Reynolds-averaged Navier-Stokes equations that describe the conservation of mass and momentum of a viscous fluid. Realizable $k-\varepsilon$ model is adopted as the turbulence model, which is suitable for the problems such as rotating flow, flow separation, and secondary flow. And the equations are as follows:

$$
\begin{gathered}
\frac{\partial v_{i}}{\partial x_{i}}=0 \\
\rho \frac{\partial v_{i}}{\partial t}+\rho \frac{\partial}{\partial x_{j}}\left(v_{i} v_{j}\right) \\
=-\frac{\partial P}{\partial x_{i}}+\frac{\partial}{\partial x_{j}}\left(\mu \frac{\partial v_{i}}{\partial x_{j}}-\rho v_{i} v_{j}\right)+\rho f_{i} \\
\frac{\partial}{\partial t}(\rho k)+\frac{\partial}{\partial x_{i}}\left(\rho k v_{i}\right) \\
=\frac{\partial}{\partial x_{i}}\left[\left(\mu+\frac{\mu t}{\sigma_{k}}\right) \cdot \frac{\partial k}{\partial x_{j}}\right]+G_{k} \\
+G_{b}-\rho \varepsilon-Y_{M}+S_{k}
\end{gathered}
$$




$$
\begin{aligned}
\frac{\partial}{\partial t}(\rho \varepsilon) & +\frac{\partial}{\partial x_{j}}\left(\rho \varepsilon v_{j}\right) \\
= & \frac{\partial}{\partial x_{j}}\left[\left(\mu+\frac{\mu t}{\sigma_{\varepsilon}}\right) \cdot \frac{\partial k}{\partial x_{j}}\right]+\rho C_{1} \cdot S_{\varepsilon}-\rho C_{2} \\
& \frac{\varepsilon^{2}}{k+\sqrt{\nu \varepsilon}}+C_{1 \varepsilon} \cdot \frac{\varepsilon}{k} \cdot C_{3 \varepsilon} \cdot G_{b}+S_{\varepsilon} .
\end{aligned}
$$

Enhanced wall function method is used near the wall. The convective terms and diffusion terms are discrete through second order windward difference format and central difference format, respectively. Physical time step length of unsteady calculation is $0.0001725 \mathrm{~s}$.

At the inlet, the boundary condition is a purely axial injection condition with uniform stagnation pressure. Classical downstream boundary conditions (such as a uniform static pressure) are not sufficient to simulate an operating point near the stall condition. When the slope of the characteristic becomes null, the calculation cannot perform the fixed downstream pressure and convergence will become difficult to achieve. A solution is to use a boundary condition with a higher number of freedom degrees, such as an idealized throttle condition that represents better the experimental facility behavior. Fan and throttle valve are linked directly. Ignoring air cavity storage effect and the inertial effect of working medium, the ventilation system operating condition is determined by the intersection of fan performance curve and throttle valve throttle line. A quadratic law is thus applied at the outlet to determine the static pressure $P s_{\text {out }}$ :

$$
P s_{\text {out }}(t)=P i_{\text {in }}+\frac{1}{2} \frac{k_{0}}{k_{1}} \rho U^{2} .
$$

The valve opening $k_{1}$ of the initial condition is set as 1 through setting the value of $k_{0}$. With the decrease of the valve opening, flow falls down and the fan gradually approaches the occurrence of rotating stall until $k_{1}$ tends to be 0 and the valve is completely closed.

3.2. Meshing Strategy. For the complex of geometric structure and flow field of the centrifugal fan, block meshing method is adopted in this paper. Hexahedral structured grid is used in inlet pipe, current collector, and outlet pipe, while for the complex flow field zones, such as impeller and volute, tetrahedral unstructured grid suits well because of its better applicability. Because of the high incidence of boundarylayer separation on blade surfaces and the significance of volute tongue on the fan performance, the boundary layers are arranged on the blade and volute surfaces. And the order of magnitudes of the distance between the wall and the first layer grid node should be within the range of $y+<1$. In order to insure the computational accuracy, mesh refinement is conducted in some critical zones such as impeller and volute tongue with the use of size function.

After the independence calculation, the grid number of the calculation model is identified as follows. The grid number of inlet section is 157000 , the grid number of impeller

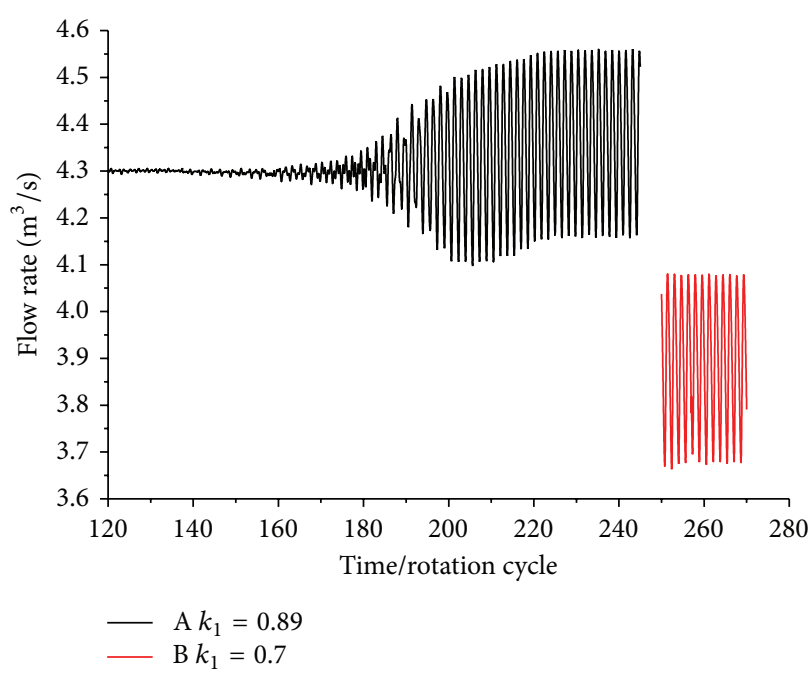

FIGURE 2: Flow rate variations with time on two valve openings.

zone is 1647800 , and the grid number of volute and outlet sections is 1204000; the total grid number of the fan is 3008800 .

3.3. Sound Field Calculation Model. The broadband noise sources model is used to conduct numerical simulation of the internal pneumatic noise source distribution of the fan in this paper. Because the noise frequency is not a fixed value, the statistical distribution of turbulence parameters, which can be obtained by solving Reynolds time-averaged equations, and acoustic analog can explain the source of broadband noise. The calculation method of acoustical power (W) and acoustic power level $\left(L_{W}\right)$ is as follows:

$$
\begin{gathered}
W=c \rho_{0}\left(\frac{u^{3}}{l}\right) \frac{u^{5}}{a_{0}^{5}} \quad(\mathrm{~W}), \\
L_{W}=10 \lg \frac{W}{W_{0}} \quad(\mathrm{~dB}) .
\end{gathered}
$$

\section{Results and Discussions}

4.1. Aerodynamic Characteristics. The full pressure of centrifugal fan with different valve opening (volume flow) obtained by numerical calculation is illustrated in Table 1 . When gradually reducing the opening of valve, the stable convergence of unsteady calculation indicates that the flow decreases and the pressure increases. When the valve opening $k_{1}$ is 0.9 , the centrifugal fan can approach a stable convergence along the throttle line without the occurrence of rotating stall. And when the valve opening $k_{1}$ is 0.89 , the full pressure can reach its maximum. With the decreasing of the valve opening, the average of full pressure gradually falls down. As can be seen, cyclical fluctuations of full pressure and flow occur since the $k_{1}=0.89$ operating point. And the fluctuation range tends to be larger with the decreasing of valve opening.

The fan flow curves when the valve opening $k_{1}$ is 0.89 and 0.7, respectively, are shown in Figure 2. According to 


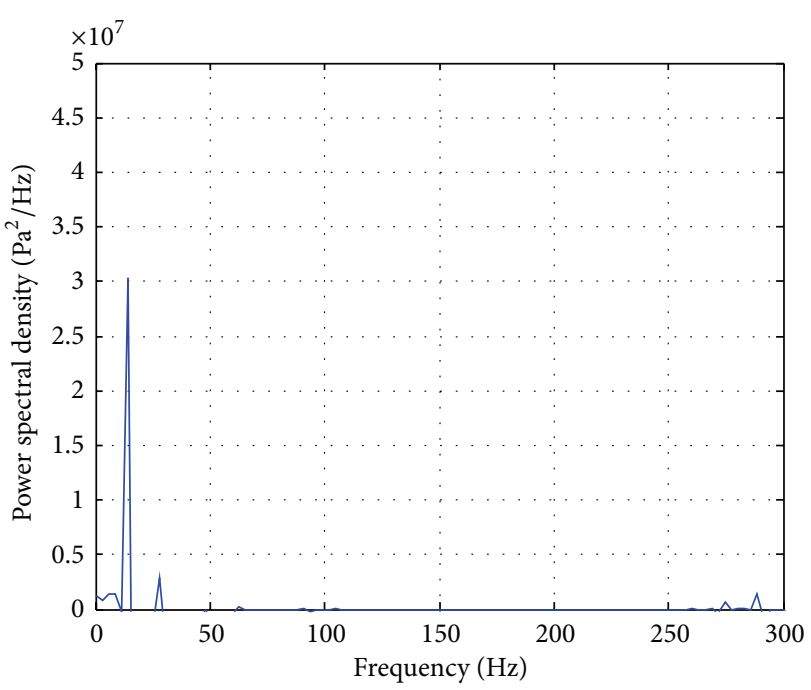

(a) $k_{1}=0.89$

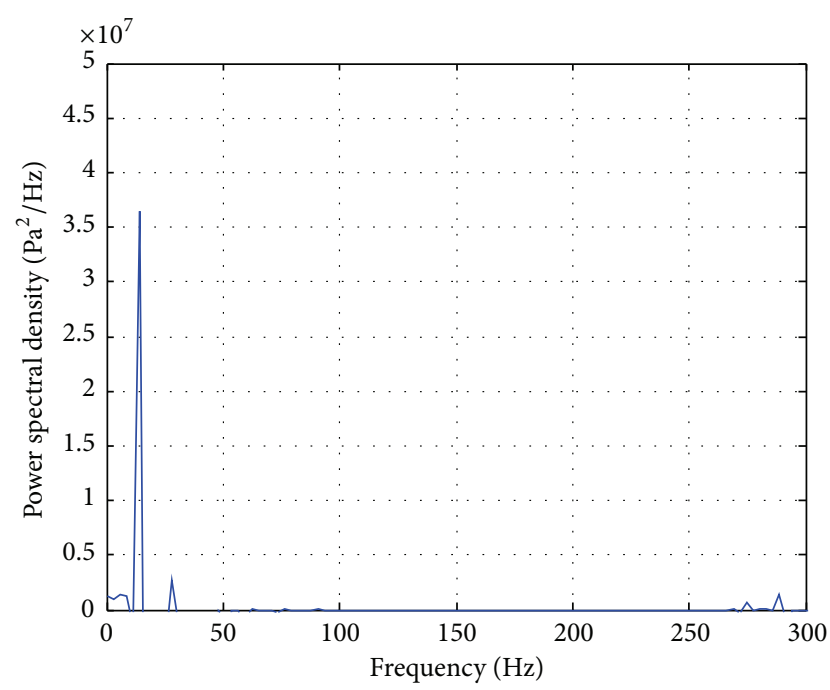

(b) $k_{1}=0.7$

FIGURE 3: Power spectrum analysis of relative speed.

TABLE 1: Relation of full pressure and flow rate.

\begin{tabular}{lcccc}
\hline $\begin{array}{l}\text { Valve } \\
\text { opening }\end{array}$ & $\begin{array}{c}\text { Volume } \\
\text { flow }\left(\mathrm{m}^{3} / \mathrm{s}\right)\end{array}$ & $\begin{array}{c}\text { Average } \\
\text { flow } \\
\left(\mathrm{m}^{3} / \mathrm{s}\right)\end{array}$ & $\begin{array}{c}\text { Full } \\
\text { pressure } \\
(\mathrm{Pa})\end{array}$ & $\begin{array}{c}\text { Average full } \\
\text { pressure } \\
(\mathrm{Pa})\end{array}$ \\
\hline 0.7 & $3.678-4.079$ & 3.877 & $1869-2292$ & 2075 \\
0.8 & $3.925-4.318$ & 4.12 & $1880-2273$ & 2076.5 \\
0.89 & $4.142-4.536$ & 4.33 & $1889-2263$ & 2080.5 \\
0.95 & 4.41 & 4.41 & 2071 & 2071 \\
1 & 4.51 & 4.51 & 2065 & 2065 \\
\hline
\end{tabular}

curve A, stall inception occurs in the 150th rotation cycle and then develops gradually with the increase of the flow fluctuation range. After 60 rotation cycles, the fan flow fluctuation amplitude gets stabilized. Then the flow fluctuates in a stable range with fixed frequency, and the fan comes into stable rotating stall at the 230 th rotation cycle. When the valve opening is decreased from 0.89 to 0.7 , as shown in curve $B$, the cycle of flow fluctuation remains the same and the amplitude slightly increases compared with curve A.

4.2. Frequency Domain Characteristics. Fast Fourier transform and the power spectrum analysis for the velocity data acquired at the entrance of the impeller are conducted under the $k_{1}=0.89$ and $k_{1}=0.7$ conditions. As shown in Figure 3, in the two valve openings, there are three isolated frequencies of $14.15 \mathrm{~Hz}, 28.31 \mathrm{~Hz}$, and $290 \mathrm{~Hz}$, wherein $14.15 \mathrm{~Hz}$ is the stall frequency and $28.31 \mathrm{~Hz}$ is the second order frequency of stall frequency. Since the impeller speed is $1450 \mathrm{rpm}$ and the leaf number is $12,290 \mathrm{~Hz}$ is the base frequency of blade passing frequency, which is quite small compared to stall frequency, indicating the fan enters deep stall.

When the valve opening is 0.7 , stall frequency shown in Figure $3(\mathrm{~b})$ is the same as that when $k_{1}=0.89$. Therefore, the flow variation has no effect on stall frequency and stall

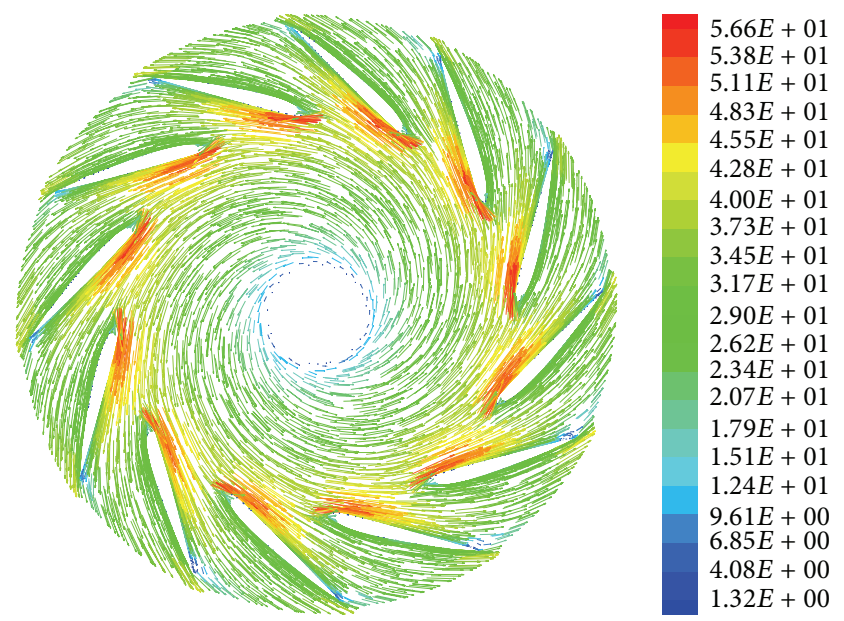

FIgURE 4: Vectors of relative speed of impeller in design condition.

cell propagation velocity. Once the stall cell is completely formed, the propagation velocity remains constant when the flow decreases. It can also be found from the figure that, along with the decrease of flow rate, the amplitude of the power spectral density increases; namely, the energy of stall cells is enhanced as the flow rate decreases.

Under the same operating conditions, the stall frequency obtained from experiment is $14.4 \mathrm{~Hz}$ [10], which is consistent with the calculation results, verifying the correctness of the numerical simulation.

4.3. Analysis of Flow Field Characteristics. Three operating conditions including design condition and $k_{1}=0.89$ and $k_{1}=0.7$ conditions are selected to study the flow field characteristics in centrifugal fan. The relative velocity vector distributions on $Z=30, Z=38.5$, and $Z=46$ axial sections with each operating condition are analyzed. 


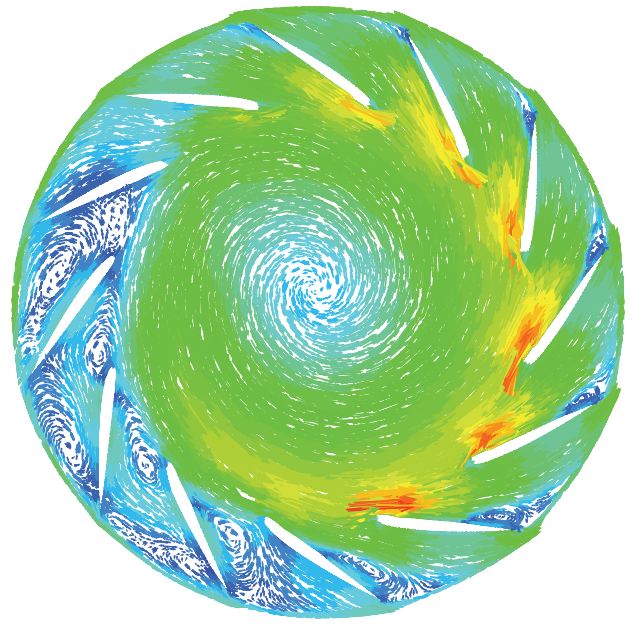

(a) $Z=30$
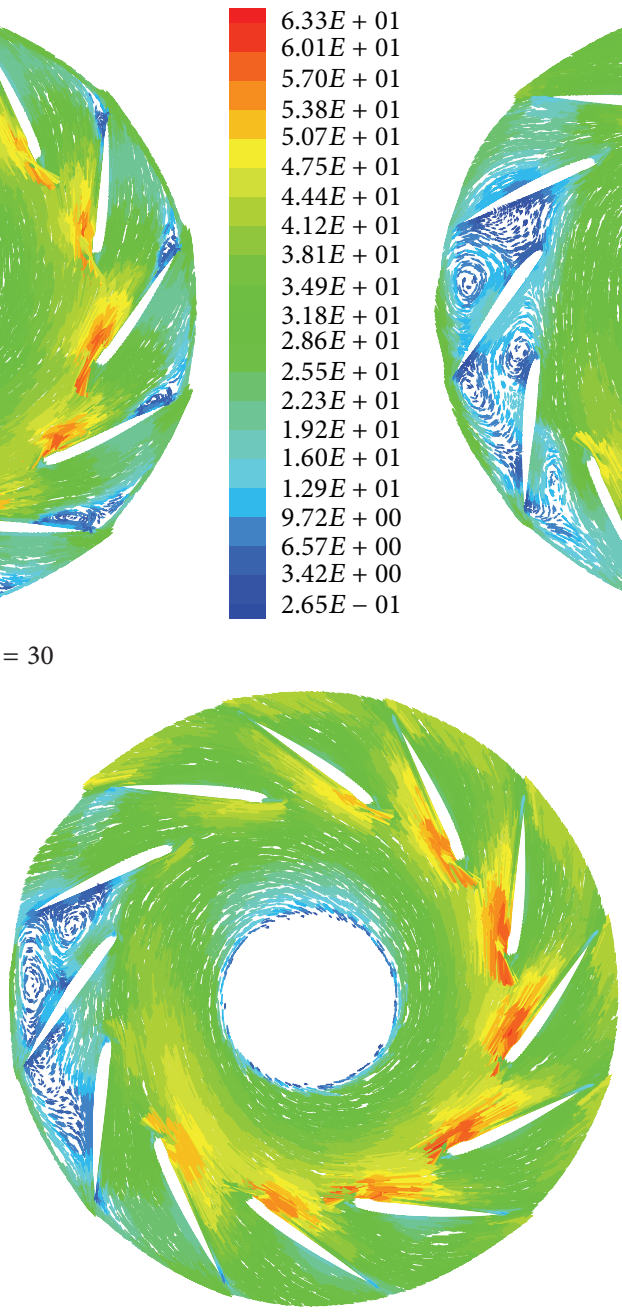

(c) $Z=46$

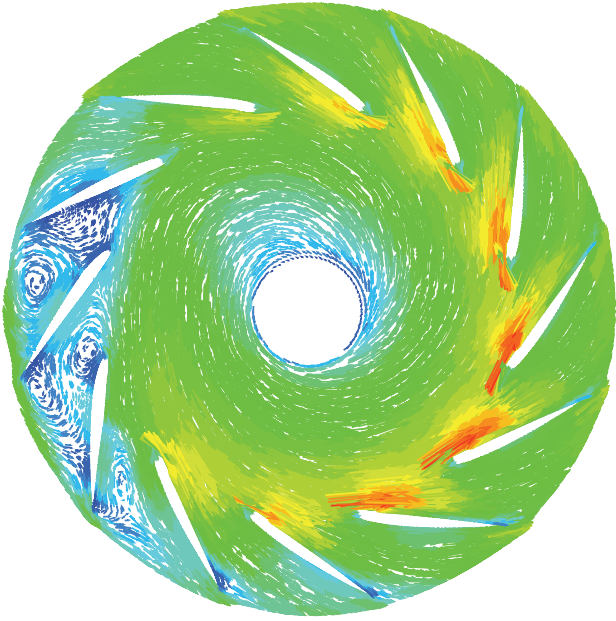

$5.90 E+01$ $5.61 E+01$ $5.31 E+01$ $5.02 E+01$ $4.73 E+01$ $4.43 E+01$ $4.14 E+01$ $3.85 E+01$ $3.55 E+01$ $3.26 E+01$ $2.97 E+01$ $2.68 E+01$ $2.38 E+01$ $2.09 E+01$

$1.80 E+01$ $1.50 E+01$ $1.21 E+01$ $9.17 E+00$ $6.24 E+00$ $3.30 E+00$ $3.73 E-01$

(b) $Z=38.5$

FIGURE 5: Vectors of relative speed of impeller at different axial cross sections when $k_{1}=0.89$.

The relative velocity vector of the $Z=38.5$ section in the impeller under design condition is illustrated in Figure 4. It can be seen that flow in each passage is relatively uniform in the same direction, and counterclockwise prewhirl exists from the hub to the impeller inlet. The flow is along the blade tangential due to the small blade entrance angle. There is no vortex structure in the impeller, and a typical jet-wake structure exists in the impeller outlet. Because of the boundary-layer separation at the trailing edge of blade suction side, the flow speed is low, and the relative speed increases from the suction side to the pressure side until the maximum.

Figure 5 shows the velocity vector distribution of different axial sections during deep stall when the valve opening $k_{1}$ is 0.89 . It can be seen from the figures that the velocity vector distribution in impeller shows severe circumferential nonuniformity; the vortex shedding near the front disc leads to flow skewness in multiple channels, resulting in the improvement in the other passages during stable stall. The stall cells rotate along the circumference direction, and the influence region towards the impeller inlet continuously increases until a steady state. Even radial countercurrent appears from the blade outlet to the blade inlet in some passages. The influence area of stall cells in the circumferential direction is consolidated and it further extends to the inlet direction according to Figure 5(b). The same position in the two flow passages is completely blocked in Figure 5(c).

Figure 6 shows the velocity vector distribution of different axial sections during deep stall when the valve opening $k_{1}$ is 0.7. Compared to Figure 5, the distribution of stall cell is similar to that when $k_{1}$ is 0.89 , and with the turning down of valve opening, the influence area of stall cells in the circumferential direction gets enlarged. The comparison between Figure 5(c) and Figure 6(c) shows that at $Z=30$ section near the front disc and the $Z=38.5$ section near the half blade height when $k_{1}$ is 0.7 , the areas occupied by stall cells both increase. At the $Z=46$ section near the rear disc, the number of passages completely blocked by stall cells increases to 4 and the blocking region expands significantly. 


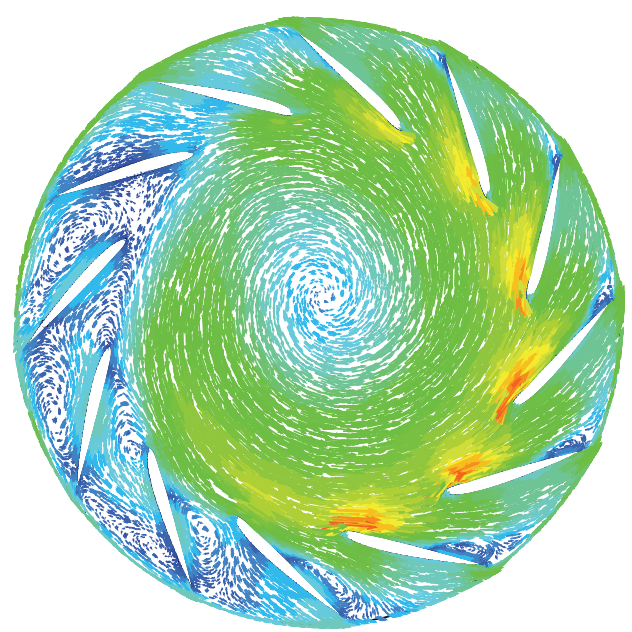

(a) $Z=30$
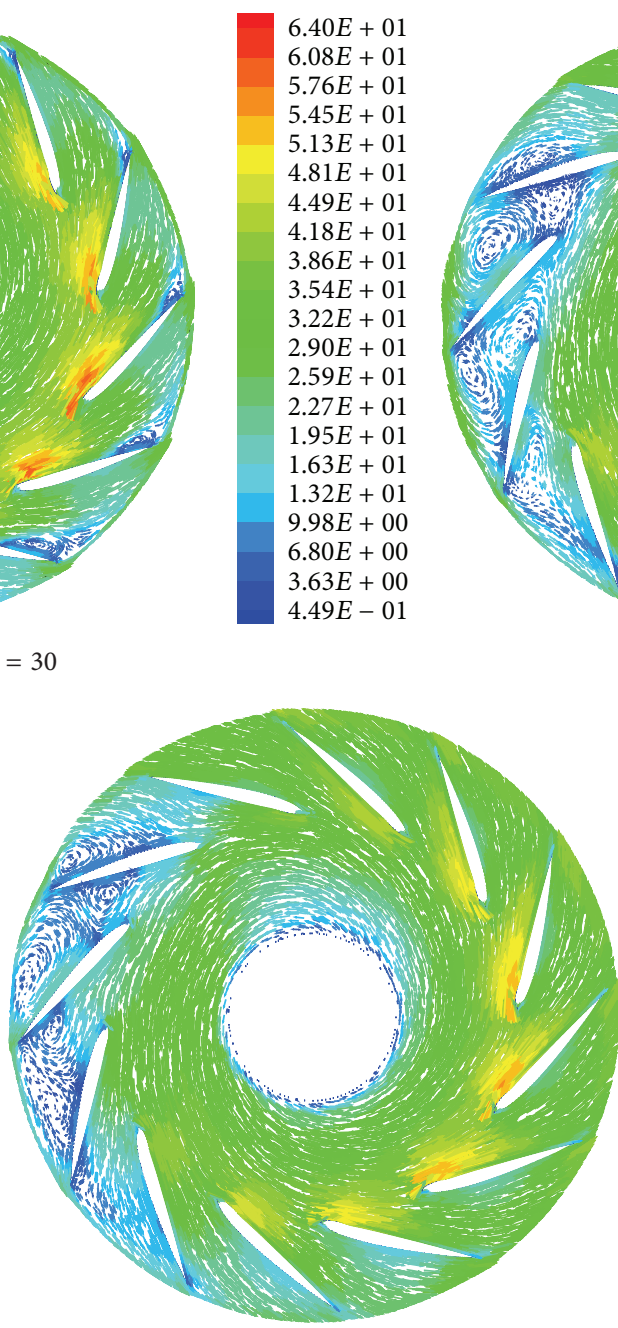

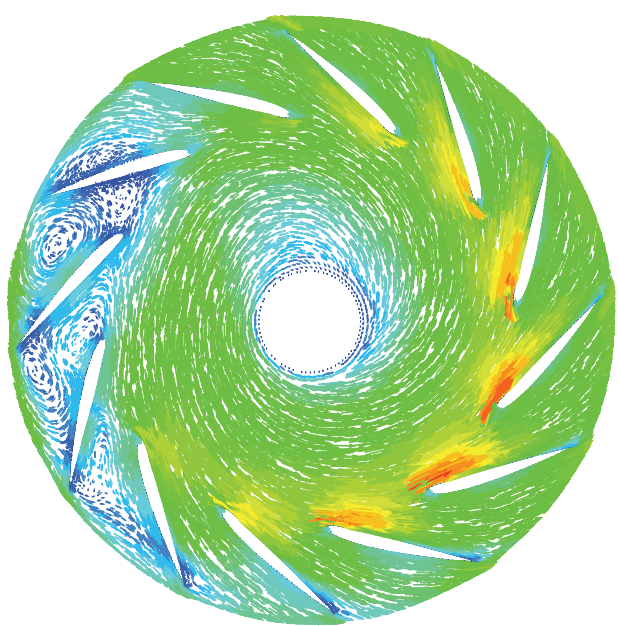

(b) $Z=38.5$
$5.99 E+01$

$5.69 E+01$

$5.39 E+01$

$5.10 E+01$

$4.80 E+01$

$4.50 E+01$

$4.20 E+01$

$3.91 E+01$

$3.61 E+01$

$3.31 E+01$

$3.01 E+01$

$2.72 E+01$

$2.42 E+01$

$2.12 E+01$

$1.82 E+01$

$1.52 E+01$

$1.23 E+01$

$9.30 E+00$

$6.32 E+00$

$3.34 E+00$

$3.65 E-01$

(c) $Z=46$

FIGURE 6: Vectors of relative speed of impeller at different axial cross sections when $k_{1}=0.7$.

4.4. Noise Characteristics Analysis. The noise of the centrifugal fan mainly comes from aerodynamic noise, mechanical noise, and electromagnetic noise, wherein the aerodynamic noise, including rotation noise and vortex noise, accounts for the major part of the total noise and is most difficult to control. In order to study the influence of rotating stall on the aerodynamic noise, three operating conditions including design condition and $k_{1}=0.89$ and $k_{1}=0.7$ conditions are selected, and contours of sound power level under the three conditions are shown in Figure 6. It can be seen from the figure that the maximum of sound power level all appears in the tongue region under three conditions. This is because periodic pressure and velocity fluctuations, which can produce high rotation noise, will occur in the volute tongue region when blade channels pass volute tongue. In addition, the noise level distribution in the volute reflects the broadband noise due to flow separation.

The following discussions mainly focus on the influence of rotating stall on the sound power level distribution under the three different conditions. Figure 7(a) shows that, under design condition, the sound power level at the blade inlet is larger, and vortex noise is generated due to flow separation here. There are 12 high noise regions with approximately $72 \sim 86 \mathrm{~dB}$ noise level uniformly distributed along the circumferential direction at the impeller outlet. This is because there is a small area of boundary-layer separation at the impeller outlet and the velocity gradient is relatively large. It can also be observed that the lowest noise level occurs at the region near the hub.

Contour of sound power level of the fan under rotating stall when $k_{1}$ is 0.89 is illustrated in Figure 7(b). The figure shows that the sound power level distribution of the impeller region along the circumferential direction is uneven, and high noise appears in 3 consecutive impeller channels with a noise reduction in other impeller channel outlets. As shown in Figure 5(b), since three channels are occupied by the stall cell, separation vortex and flow blockage occur in the channels, resulting in high noise, while in other impeller channels, flow rate increases and incidence angles decrease, resulting in flow improvement and vortex noise reduction. 


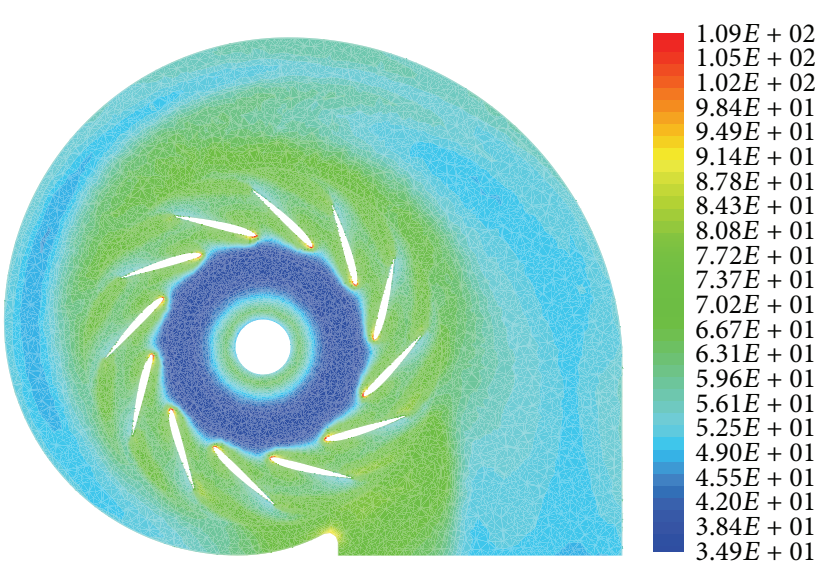

(a) Design flow rate

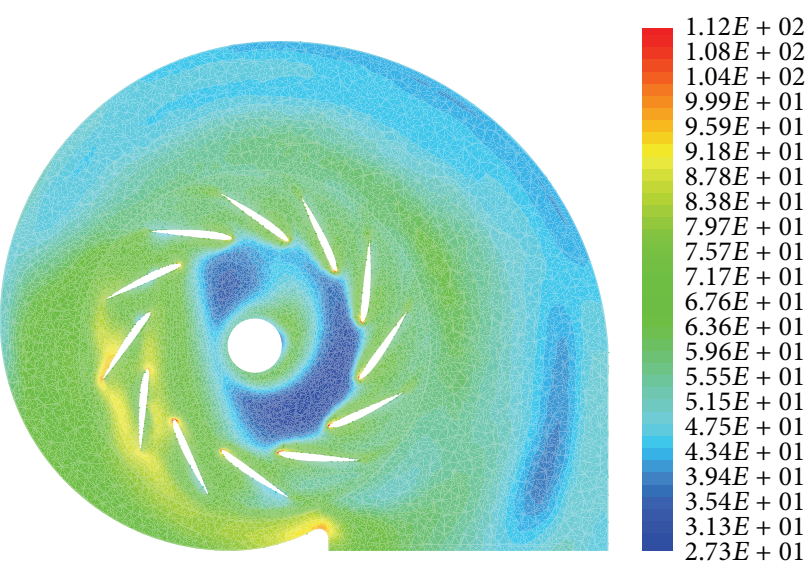

(b) $k_{1}=0.89$

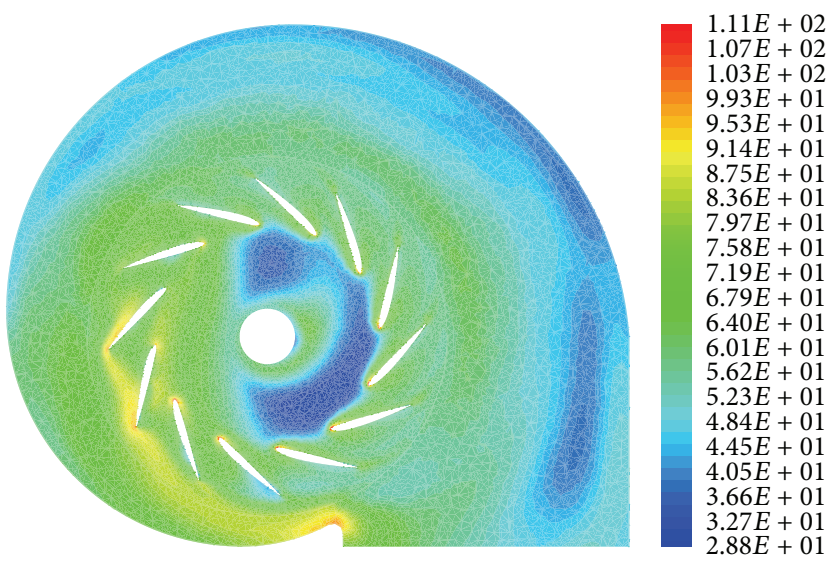

(c) $k_{1}=0.7$

FIGURE 7: Contours of sound power level in different operation conditions.

Besides, high noise areas rotate with the stall cells in the same speed along the circumferential direction. And the low noise area near the hub reduces by $1 / 4$ due to the influence of stall cells.

Contour of sound power level of the fan under rotating stall when $k_{1}$ is 0.7 is illustrated in Figure $7(\mathrm{c})$. With the decrease of valve opening, the affected regions of stall cells expand circumferentially. Currently, four impeller channels are occupied by stall cells (Figure 6(c)), and the interaction of stall and volute tongue leads to belt-shaped high noise areas near the impeller outlet. Along with the circumferential propagation of stall cells, the high noise areas also show circumferential unsteady change, and the low noise areas in the hub region continue to decrease to approximately $1 / 3$ of that in design condition.

Based on the analysis above, it indicates that the rotation noise near the volute tongue accounts for the major part of fan noise under design condition. Once flow decreases and fan enters rotating stall, the vortex noise at the impeller outlet accounts for the major part of fan noise, and the high noise area rotates circumferentially with the stall cells. Under the three conditions, with the decrease of flow, the maximum of the sound power level of the fan gradually increases, reflecting the great importance of rotating stall on the fan noise under low flow rate conditions.

4.5. Noise Characteristics during Circumferential Propagation of Stall Cells. The contours of sound power level at the $Z=38.5$ section of fan are illustrated in Figure 8 . At all 4 moments, the fan is in deep stall and the valve opening $k_{1}$ is 0.89 . The time interval of each moment is $1 / 12$ rotation cycle, and the impeller rotates along the clockwise direction. It can be seen from Figure 8(a) that the high noise areas are mainly concentrated in the volute tongue area and three consecutive impeller passages nearby. This is because the stall cell occupies the corresponding three impeller passages and, as a result, high vortex noise is generated, while in the other impeller passages, due to the improvement of flow, sound power level is low and evenly distributed. Meanwhile, the stall cell propagates to the volute tongue, resulting in stronger velocity and pressure fluctuations. A high volute tongue noise is also induced. Under the superimposing effect of the volute tongue noise and the vortex noise caused by stall cell, the high noise area develops into a noise area with a higher sound power level. 


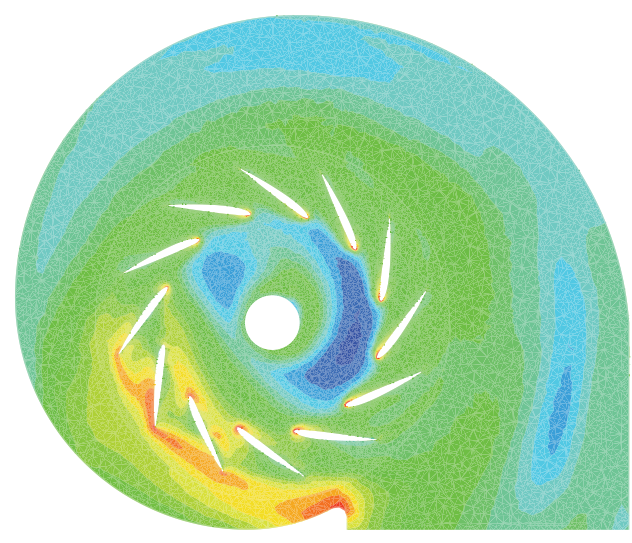

(a) The first moment

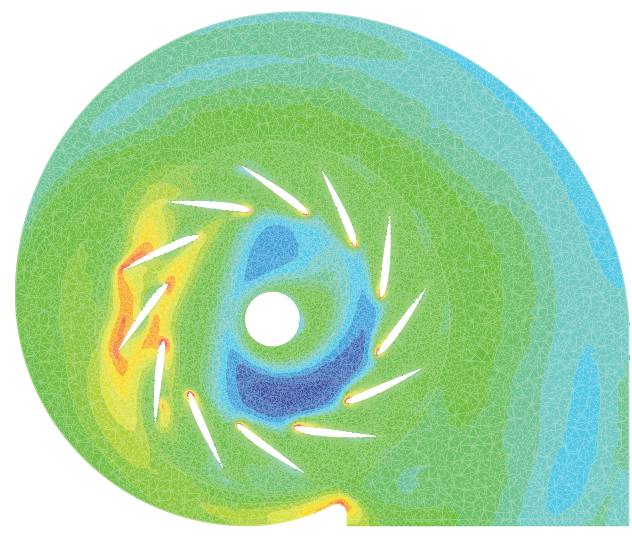

(c) The third moment

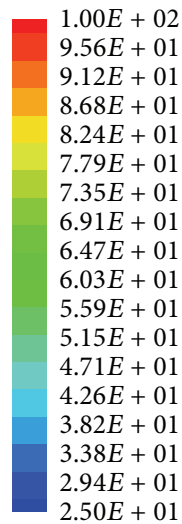

$2.50 E+01$

$1.00 E+02$ $9.56 E+01$ $9.12 E+01$ $8.68 E+01$ $8.24 E+01$ $7.79 E+01$ $7.35 E+01$ $6.91 E+01$ $6.47 E+01$ $6.03 E+01$ $5.59 E+01$ $5.15 E+01$ $4.71 E+01$ $4.26 E+01$ $3.82 E+01$ $3.38 E+01$ $2.94 E+01$ $2.50 E+01$

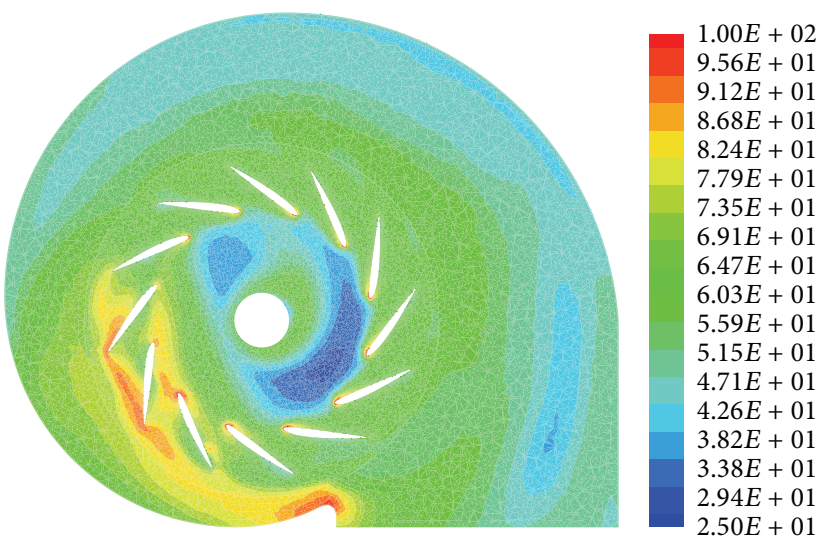

(b) The second moment

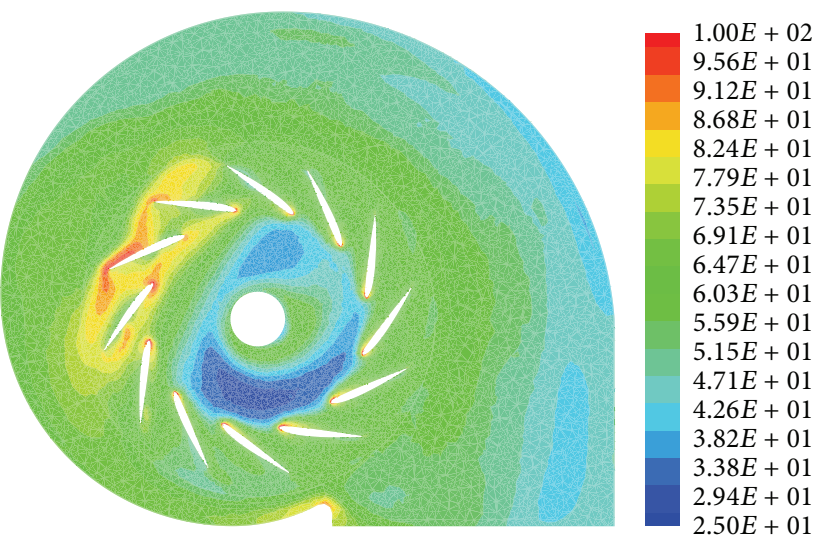

(d) The fourth moment

FIGURE 8: Contours of sound power level in stall condition.

As shown in Figure 8(b), under the combining influence of both stall cell and volute tongue, the high noise area is gradually elongated. Due to the propagation of stall cell, it gradually gets away from the area of volute tongue, resulting in weakening the superimposing effect. As time goes by, the high noise area in Figure 8(c) gets further elongated with a trend of separation and the sound power level of high noise areas decreases. In Figure 8(d), the high noise areas corresponding to the vortex noise and volute tongue noise basically separate. And the sound power level corresponding to volute tongue greatly declines.

It can be drawn from Figure 8 that while the impeller passes three passages along clockwise direction, the high noise area passes two impeller passages along the clockwise direction. It indicates that, in the absolute coordinate reference system, the high noise area occupying about three impeller passages rotates in the same direction with impeller under rotating stall. It also has the same speed with stall cells, while in the relative coordinate reference system, high noise area spreads in the opposite direction of the rotation of the impeller.

Through the analysis above, there are two major sources of noise in a centrifugal fan under rotating stall, namely, the vortex noise caused by stall and the volute tongue noise caused by the rotation of impeller. When the stall cell spreads to the volute tongue, due to the superimposing effect of vortex noise and volute tongue noise, the sound power level is the highest and the high noise area is the largest. While the stall cell is away from the volute tongue, the corresponding high noise areas separate gradually. Along with that, the sound power level decreases and the high noise area becomes smaller. Therefore, the aerodynamic noise of the centrifugal fan under rotating stall changes periodically over time, and the fluctuation period is the same with the rotating period of the stall cell.

\section{Conclusions}

A 3D unsteady RANS method has been used to compute the flow in the full annulus of a centrifugal fan with the objective of investigating the influence of rotating stall on flow field and aerodynamic noise at different operating points. The following points summarize this study.

(1) During stall conditions, with the turning down of valve opening, the flow and full pressures fall down and the amplitude of fluctuation tends to be larger. The decrease of flow rate shows no influence on the stall frequency but makes the corresponding power 
spectral density larger; namely, it enhances the energy of stall cells.

(2) Under design condition, the flow in each passage is relatively uniform. During stall condition, the velocity vector distribution in impeller shows circumferential nonuniformity. About 2 impeller passages are completely blocked with the separated vortex in stall condition. Meanwhile, the flow in other impeller passages is improved with the increase of flow rate in these passages. With the decrease of valve opening from 0.89 to 0.7 , the flow field in the impeller tends to be worse and the number of passages completely occupied by the stall cells increases from 2 to 4 .

(3) Under design condition, the rotation noise near the volute tongue accounts for the major part of fan noise. With decrease of flow rate, the fan enters stall conditions and the vortex noise at the impeller outlet accounts for the major part of fan noise. The high noise area rotates circumferentially with the stall cell. With the decrease of flow rate, the maximum of the sound power level of the fan gradually increases, reflecting the great influence of stall cell on the fan noise under low flow conditions.

\section{Conflict of Interests}

The authors declare that they have no financial or personal relationships with other people or organizations that can inappropriately influence their work; there is no professional or other personal interests of any nature or kind in any product, service, and/or company that could be construed as influencing the position presented in, or the review of, this paper.

\section{Acknowledgments}

The authors gratefully acknowledge the support provided to this research by the National Natural Science Foundation of Hebei Province under Grant no. E2012502016 and the Fundamental Research Funds for the Central Universities under Grant no. 12QN39.

\section{References}

[1] N. Gourdain, S. Burguburu, F. Leboeuf, and G. J. Michon, "Simulation of rotating stall in a whole stage of an axial compressor," Computers \& Fluids, vol. 39, no. 9, pp. 1644-1655, 2010.

[2] E. Lennemann and J. H. G. Howard, "Unsteady flow phenomena in rotating centrifugal impeller passages," Journal of Engineering for Gas Turbines and Power, vol. 92, no. 1, pp. 65-72, 1970.

[3] A. Lucius and G. Brenner, "Numerical simulation and evaluation of velocity fluctuations during rotating stall of a centrifugal pump," Journal of Fluids Engineering, vol. 133, no. 8, Article ID 081102, 8 pages, 2011.

[4] V. Hasmatuchi, M. Farhat, S. Roth, F. Botero, and F. Avellan, "Experimental evidence of rotating stall in a pump-turbine at off-design conditions in generating mode," Journal of Fluids Engineering, vol. 133, no. 5, Article ID 051104, 8 pages, 2011.
[5] C. Rodgers, "A diffusion factor correlation for centrifugal impeller stalling," Journal of Engineering for Gas Turbines and Power, vol. 100, no. 4, pp. 592-603, 1978.

[6] K. B. Abidogun, "Effects of vaneless diffuser geometries on rotating stall," Journal of Propulsion and Power, vol. 22, no. 3, pp. 542-549, 2006.

[7] T. R. Camp and I. J. Day, "A study of spike and modal stall phenomena in a low-speed axial compressor," Journal of Turbomachinery, vol. 120, no. 3, pp. 393-401, 1998.

[8] D. C. Leinhos, N. R. Schmid, and L. Fottner, "The influence of transient inlet distortions on the instability inception of a low-pressure compressor in a turbofan engine," Journal of Turbomachinery, vol. 123, no. 1, pp. 1-8, 2001.

[9] M. Choi, M. Vahdati, and M. Imregun, "Effects of fan speed on rotating stall inception and recovery," Journal of Turbomachinery, vol. 133, no. 4, Article ID 041013, 8 pages, 2011.

[10] L. Zhang, S. L. Wang, Q. Zhang, and Z. R. Wu, "Dynamic characteristics of rotating stall for centrifugal fans," Proceedings of the CSEE, vol. 32, no. 14, pp. 95-102, 2012.

[11] M. J. Lighthill, "Sound Generated Aerodynamically," Proceedings of the Royal Society A: Mathematical, Physical \& Engineering Sciences, vol. 267, no. 1329, pp. 147-182, 1962.

[12] A. K. M. Díaz, O. J. M. Fernández, E. B. Marigorta, and C. S. Morros, "Numerical prediction of tonal noise generation in an inlet vaned low-speed axial fan using a hybrid aeroacoustic approach," Journal of Mechanical Engineering Science, vol. 223, no. 9, pp. 2081-2098, 2009.

[13] C. Scheit, B. Karic, and S. Becker, "Effect of blade wrap angle on efficiency and noise of small radial fan impellers-a computational and experimental study," Journal of Sound and Vibration, vol. 331, no. 5, pp. 996-1010, 2012.

[14] A. Gérard, A. Berry, and P. Masson, "Control of tonal noise from subsonic axial fan. Part 2: active control simulations and experiments in free field," Journal of Sound and Vibration, vol. 288, no. 4-5, pp. 1077-1104, 2005.

[15] H. Ouyang, J. Tian, Y. Li, Z. Zheng, and Z. Du, "Internal flow and noise investigations about the cross-flow fan with different blade angles," Journal of Turbomachinery, vol. 134, no. 5, Article ID 051023, 9 pages, 2012.

[16] D.-J. Lee, W.-H. Jeon, and K.-H. Chung, "Development and application of fan noise prediction method to axial and centrifugal fan," in Proceedings of the ASME Joint U.S.-European Fluids Engineering Division Conference, vol. 2, pp. 987-992, Montreal, Canada, July 2002. 


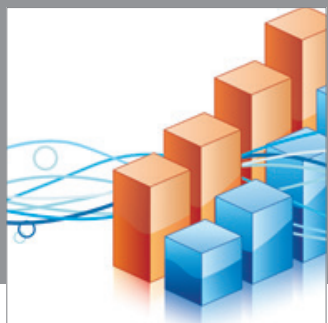

Advances in

Operations Research

mansans

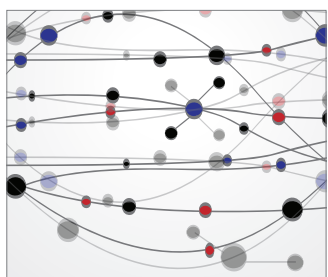

The Scientific World Journal
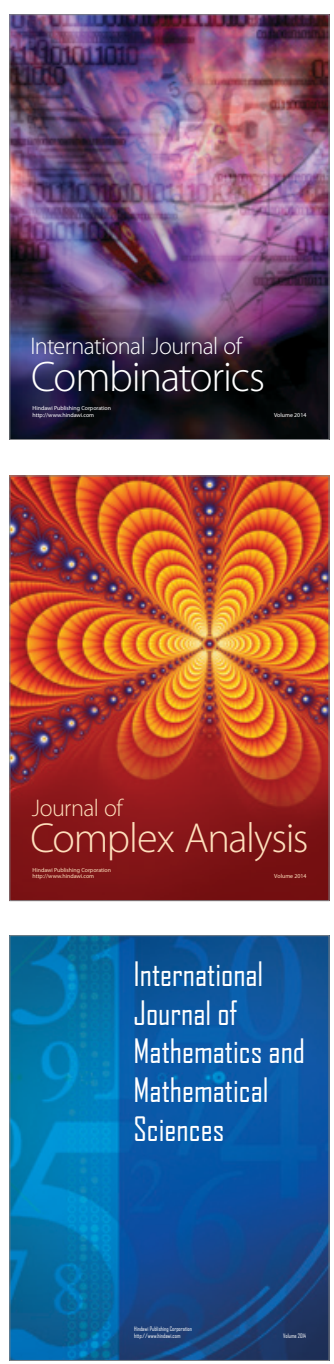
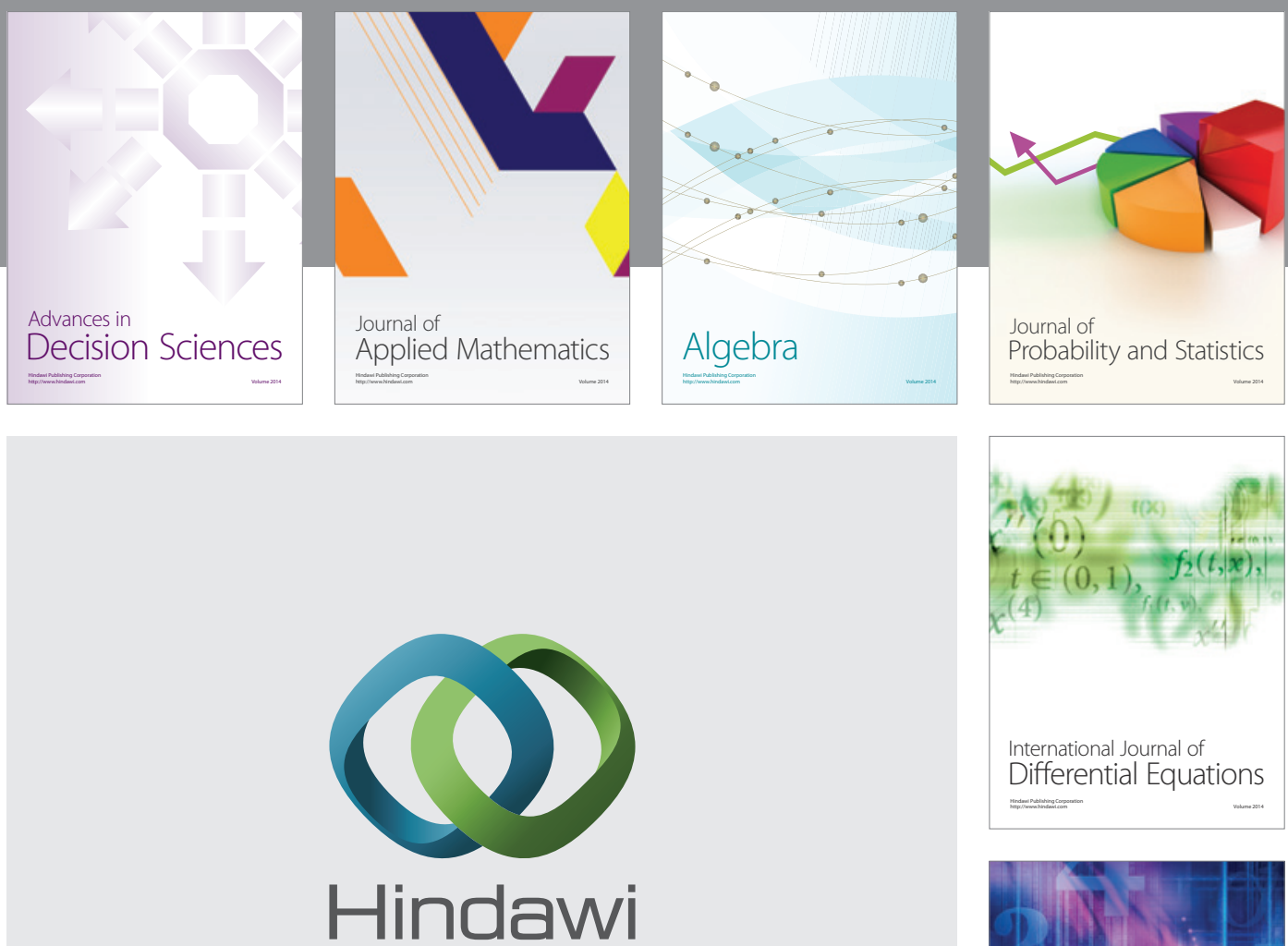

Submit your manuscripts at http://www.hindawi.com
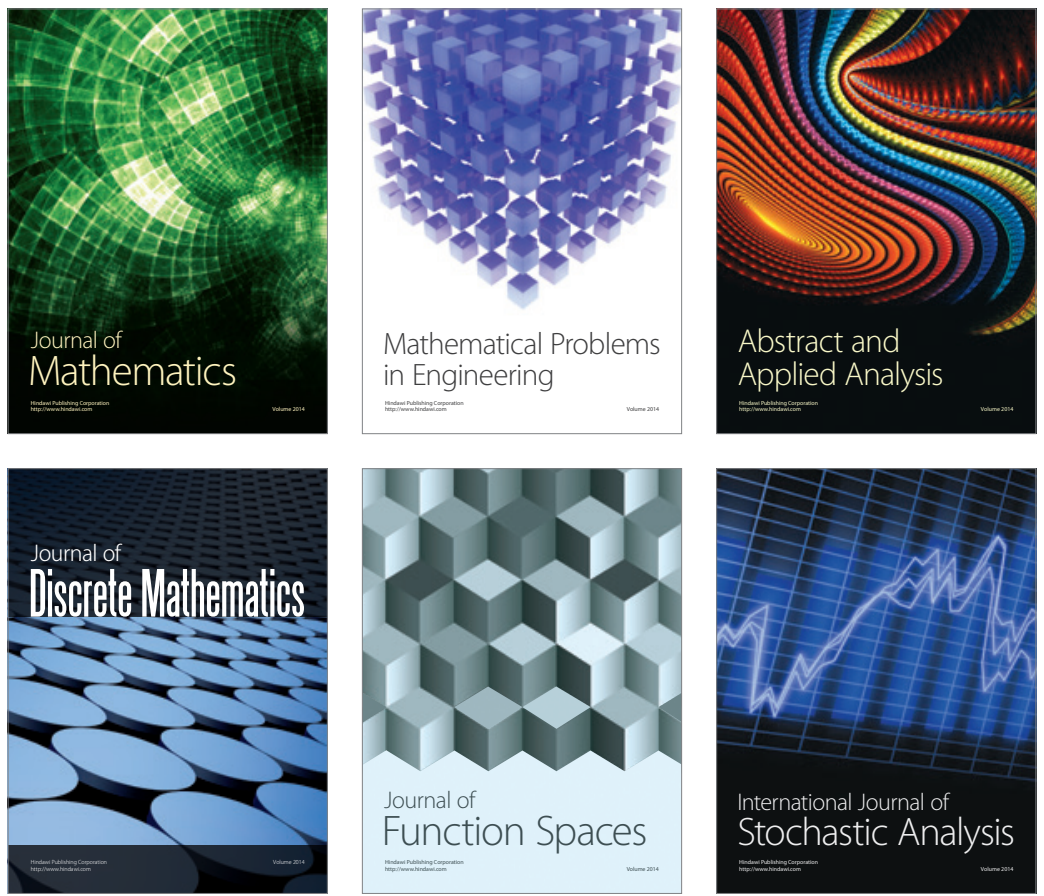

Journal of

Function Spaces

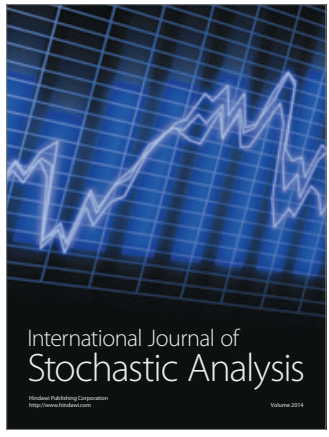

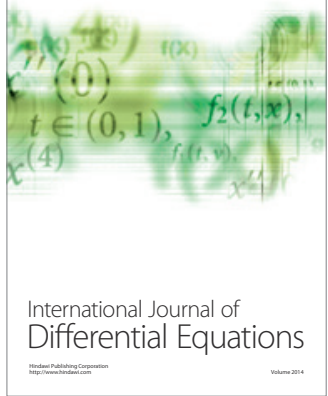
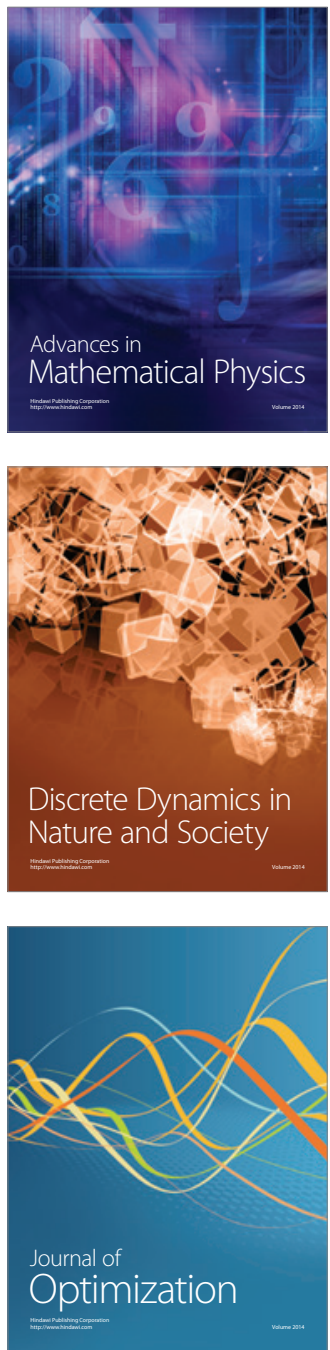